\title{
Frequent gain of the human telomerase gene TERC at $3 q 26$ in cervical adenocarcinomas
}

\author{
S Andersson', K-L Wallin², A-C Hellström ${ }^{3}$, LE Morrison ${ }^{4}$, A Hjerpe ${ }^{5}$, G Auer ${ }^{5}$, T Ried ${ }^{6}$, C Larsson ${ }^{*, 2}$ and \\ K Heselmeyer-Haddad ${ }^{6}$
}

'Department for Clinical Science, Intervention and Technology, Division of Obstetrics and Gynecology, Karolinska University Hospital-Huddinge, Karolinska Institutet, Stockholm SE-I 4 I 86, Sweden; '2Department of Molecular Medicine and Surgery, Karolinska Institutet, Karolinska University Hospital-Solna, CMM L8:0, Stockholm SE-I 71 76, Sweden; ${ }^{3}$ Department of Gynecologic Oncology, Radiumhemmet, Karolinska University Hospital-Solna, Stockholm SE171 76, Sweden; ${ }^{4}$ Abbott Molecular, 1300 East Touhy Avenue, Des Plaines, IL 600 I8, USA; ${ }^{5}$ Department of Oncology-Pathology, Karolinska University Hospital Solna, CCK, Stockholm SE-I 7 I 76, Sweden; ${ }^{6}$ Genetics Branch, Center for Cancer Research, National Cancer Institute/NIH, Building 50, Room 1408, 50 South Drive, Bethesda, MD 20892-8010, USA

The level of genomic amplification of the human telomerase gene TERC, which maps to chromosome band 3q26, was determined in primary cervical adenocarcinomas. Interphase nuclei prepared from archival material of 12 primary cervical adenocarcinomas, eight of which were human papillomavirus positive, were hybridised with a triple colour probe set specific for centromeres of chromosomes 3 and 7 and the TERC gene. We observed high proportions of nuclei with increased absolute copy numbers for TERC in all tumours (mean 3.3; range 2.3-5.2). Amplification of the human telomerase gene TERC is a consistent aberration in cervical adenocarcinomas. Therefore, application of our probe set may provide an objective genetic test for the assessment of glandular cells in Pap smears and hence for the diagnosis of cervical adenocarcinomas.

British Journal of Cancer (2006) 95, 331 -338. doi:I0.1038/sj.bjc.6603253 www.bjcancer.com

Published online 18 July 2006

(c) 2006 Cancer Research UK

Keywords: cervical adenocarcinoma; human papillomavirus; interphase fluorescence in situ hybridisation; chromosome arm 3q; telomerase; early detection

Cervical cancer is a disease that mainly affects women in the forth or fifth decade of life. According to the International Agency for Research on Cancer (IARC), it is the second most common cancer in women worldwide, and in developing nations it is the most common cancer in women (Parkin et al, 2001). Two main types of cervical cancer are described, that is, squamous cell carcinoma and adenocarcinoma. Cervical adenocarcinomas comprise $20 \%$ of all cervical cancers that are diagnosed (Vizcaino et al, 1998). Importantly, in the last few decades the incidence of cervical adenocarcinoma among young women has been steadily increasing (Vizcaino et al, 1998; Bergström et al, 1999; Smith et al, 2000; Sasieni and Adams, 2001); however, the reasons for this increase are presently unknown. Applications of clinical screening programmes that have led to a decrease in squamous cell carcinoma have only shown a limited effect on the occurrence of adenocarcinomas. This is owing to the fact that the sensitivity for detecting precursor lesions of adenocarcinomas is much lower than for the detection of precursor cells of squamous carcinomas. This can be partly explained by the difficulty in the morphological assessment of highly differentiated glandular cells, which can be derived from adenocarcinomas of the endocervix. In addition to these

*Correspondence: Dr C Larsson;

E-mail: catharina.larsson@cmm.ki.se

Received I 9 January 2006; revised I 5 May 2006; accepted 12 June 2006; published online 18 July 2006 difficulties, the endocervix is less accessible for cytological sampling (Bergström et al, 1999).

Epidemiological and molecular biological studies have shown that infection with high-risk human papillomavirus (HPV) is an important aetiological agent in the pathogenesis of cervical adenocarcinoma (Bosch et al, 1995; Bosch and Munoz, 2002). More than $99 \%$ of squamous cell carcinomas harbour oncogenic types of HPV, with HPV 16 being the most frequently encountered (Walboomers et al, 1999). Partly in contrast, we and others have found HPV DNA in 30-90\% of cervical adenocarcinomas, with HPV 18 as the predominant type (Tenti et al, 1996; Andersson et al, 2003a, b; Clifford et al, 2003; Zielinski et al, 2004; Castellsague et al, 2006).

Although tightly linked to cervical cancer development, HPV infection alone is not sufficient for oncogenic transformation, and other factors than HPV are required for the malignant transformation towards cervical adenocarcinomas (Skyldberg et al, 1999; Pirog et al, 2000). The application of cytogenetic and molecular cytogenetic techniques to study genomic alterations have identified frequent gains involving the long arm of chromosome 3 (Yang et al, 2001). This observation is in concordance with previous findings of recurrent $3 q$ amplification in squamous cell carcinomas (Heselmeyer et al, 1996, 1997a). The value of detection of genomic amplification of $3 q$ as a biomarker for progression during uterine tumorigenesis was therefore evaluated in several studies. The results showed that amplification of TERC is almost invariably found in Pap smears assessed as HSIL (i.e., high squamous intraepithelial lesions). In addition, a more recent study un- 
ambiguously established the central role of $3 \mathrm{q}$ for progression from low-grade dysplastic lesions to higher grades and to invasive carcinomas and showed that the gain of $3 \mathrm{q}$ can occur in morphologically normal Pap smears of women who developed cervical carcinomas after only a short latency (Heselmeyer-Haddad et al, 2003, 2005). Gain of chromosomal region $3 \mathrm{q}$ is also recurrently observed in other types of cancer, including tonsillar cancer and carcinomas of the anogenital region that are both associated with HPV DNA (Heselmeyer et al, 1997b; Dahlgren et al, 2003; Stoltzfus et al, 2005).

In this study, we have determined TERC copy number gain in cervical adenocarcinomas using interphase fluorescence in situ hybridisation (FISH) on cytospins prepared from single-cell suspensions of disintegrated archival paraffin-embedded tissue and correlated the findings to the presence of HPV infection and the clinical course.

\section{PATIENTS AND METHODS}

\section{Tumour material}

The study includes formalin-fixed paraffin-embedded tumour tissue specimens from 12 primary cervical adenocarcinomas diagnosed and surgically treated at the Karolinska University Hospital-Huddinge during 1992-2000 (Table 1). All tumour cases were identified from the Swedish Central Cancer Registry organised by the National Board of Health and Welfare. This registry includes all cases of malignant tumours diagnosed histopathologically after 1959, whereby each tumour is identified by a topographical and histopathological code. All tumour samples were collected with informed consent and approval from the local ethics committee.

The clinical information for each case is detailed in Table 1 and has been published previously (Andersson et al, 2003a). The histopathological diagnosis was based on the WHO criteria. The 12 tumours were classified as cervical adenocarcinomas and all were without any squamous cell component. Furthermore, the four HPV-negative tumours (T4, T5, T8, T9) were histopathologically re-evaluated and revealed typical morphology of cervical adenocarcinoma in agreement with the initial diagnosis. Immunostainings with vimentin and carcinoembryonic antigen (CEA) were carried out. Cases T4, T5 and T8 were strongly positive for CEA and negative for vimentin, in agreement with the common pattern for adenocarcinoma of cervical origin as reported by McCluggage et al (2002). Case T9 showed atypical staining pattern (CEA was negative and vimentin positive); however, the morphology was strongly suggestive of cervical origin as judged by several independent histopathologists. Seven tumours were well differentiated $(58 \%)$, one was moderately differentiated $(8 \%)$ and four tumours were poorly differentiated (33\%). Clinical staging was according to the International Federation of Gynecology and Obstetrics (FIGO) classification for cervical cancer (Benedet et al, 2000). At initial diagnosis, 10 tumours were classified as stage I (83\%) and two tumours as stage II (17\%). Two patients initially exhibited lymph node involvement (17\%). All patients were previously sampled for Pap smear analysis. All but one Pap smear (which was evaluated as showing signs of cellular inflammation) were assessed as normal at the time of the most recent cytological evaluation (Table 1). Re-evaluation of the Pap smears confirmed the initial diagnoses in all cases (Andersson et al, 2003a). All patients were retrospectively followed up from the time of diagnosis until June 2005, and disease recurrence and survival data recorded.

\section{HPV status}

Results from HPV screening analyses have been previously published for all cases (Andersson et al, 2003a). Briefly, the analyses were performed on extracted DNA obtained from sections of paraffin blocks, of which the preceding section had been used for morphological diagnosis. A fragment of $139-148 \mathrm{bp}$ was amplified from the L1 region with GP5 + /GP6 + primers, HPV typed by direct DNA sequencing and comparison to known HPV sequence databases using the BLAST algorithm (www.ncbi.nlm. nih.gov/BLAST). Eight of the 12 tumours were thus found to be HPV-positive; five tumours were infected with HPV 18, two with HPV 16 and one with HPV 45 (Table 1). In the remaining four tumours, HPV was not detected.

\section{Preparation of nuclei suspensions for FISH analysis}

Single-layer nuclei preparations for interphase FISH hybridisations were prepared using the Hedley method with modifications (Castro et al, 1993). A 50- $\mu \mathrm{m}$ section was cut from each of the 12 formalin-fixed paraffin-embedded tumour tissue samples. After deparaffinisation in xylene, the section was rehydrated in an ethanol series and in distilled water, and the section was disintegrated in $500 \mu \mathrm{l}$ of $0.1 \%$ protease $/ 1 \times$ PBS (Protease: Type XXIV, Bacterial, P 8038, Sigma, St Louis, MO, USA and Dulbecco's $1 \times$ PBS, Life Technologies, Rockville, MD, USA) at $45^{\circ} \mathrm{C}$ for 45-60 min. The reaction was stopped by adding $500 \mu \mathrm{l} 1 \times \mathrm{PBS}$ at room temperature. The sample was then filtered through a nylon membrane (CN 051, DAKO, Glostrup, Denmark), centrifuged and

Table I Clinical details and HPV analysis of the 12 adenocarcinomas in the study

Follow-up

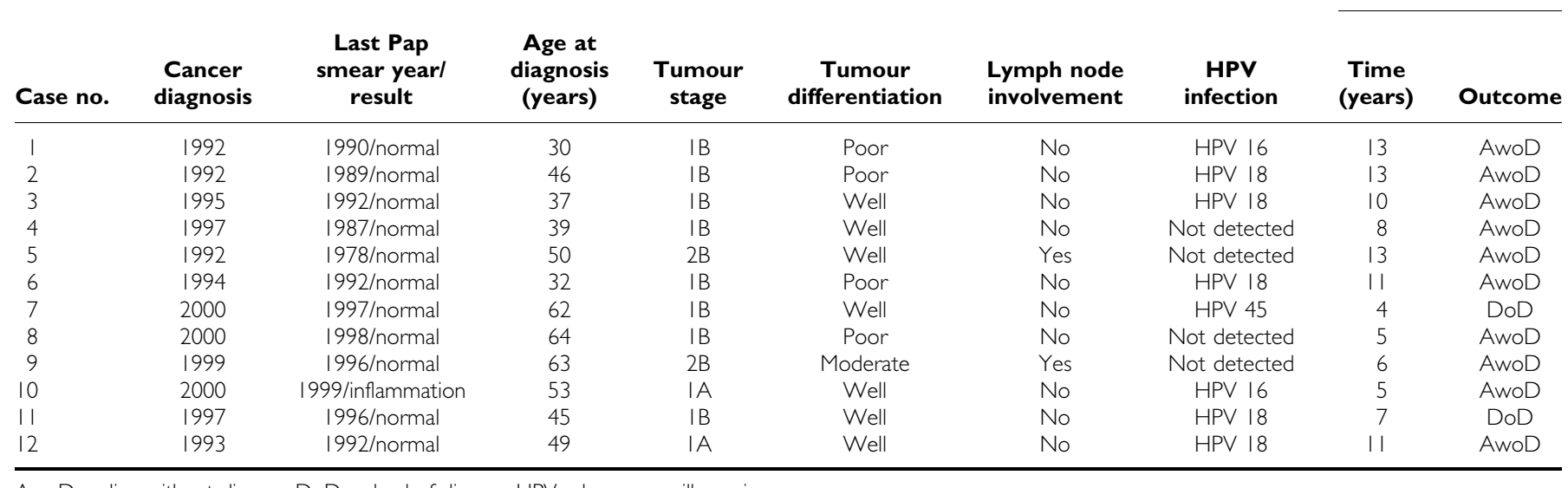

$A w o D=$ alive without disease; $\mathrm{DoD}=$ dead of disease; $\mathrm{HPV}=$ human papillomavirus. 
resuspended in $1 \times$ PBS. Cytospin slides were prepared using a Shandon Cytospin ${ }^{\circledR}$ centrifuge and fixed in an ethanol series.

\section{FISH}

Triple-colour FISH analysis was performed on each case using the following probe set: a centromere-specific probe for chromosome $7\left(\mathrm{CEP}^{\mathbb{R}} 7\right)$; a centromere-specific probe for chromosome 3 (CEP3) and a contig consisting of four overlapping BAC clones containing the TERC gene at chromosomal location 3q26. All probes were obtained from Vysis/Abbott Molecular Inc. (Des Plaines, IL, USA). The details for this probe set, its sensitivity and specificity, as well as experimental conditions were published previously (HeselmeyerHaddad et al, 2003). In short, CEP7 was labelled with Spectrum Aqua $^{\mathrm{TM}}$ (SA), CEP3 with Spectrum Green ${ }^{\mathrm{TM}}$ (SG) and the TERC contig with Spectrum Orange ${ }^{\mathrm{TM}}(\mathrm{SO})$, using chemical labelling as described (Bittner et al, 1996). Before hybridisation, the cytospin slides were pretreated with a pepsin digestion and fixed in an ethanol series. Slides were denatured in $70 \%$ formamide $/ 2 \times$ SSC for $3.5 \mathrm{~min}$ at $80^{\circ} \mathrm{C}$. The probes were denatured according to the manufacturer's recommendations. After overnight hybridisation at $37^{\circ} \mathrm{C}$, the slides were first washed four times in $50 \%$ formamide/ $2 \times \mathrm{SSC}$ at $45^{\circ} \mathrm{C}$ (once for $3 \mathrm{~min}$ and three times for $7 \mathrm{~min}$ ), followed by washes in $2 \times \mathrm{SSC}$ at $45^{\circ} \mathrm{C}$ for $5 \mathrm{~min}$ and in $2 \times \mathrm{SSC} /$ $0.1 \% \mathrm{NP} 40$ at $45^{\circ} \mathrm{C}$ for $5 \mathrm{~min}$. The slides were counterstained with 4,6-diamidino-2-phenylindole (DAPI), and subsequently embedded in an antifade solution.

\section{Scoring of FISH results}

Fluorescence in situ hybridisation and image analyses were carried out using a Leica DM-RXA fluorescence microscope (Leica, Wetzlar, Germany) equipped with custom optical filters for DAPI, SA, SG and SO (Chroma Technologies, Brattleboro, VT, USA) and $\times 40$ Plan Apo (NA 1.25) objective. Images were taken in areas of optimal cell density with minimal cellular clumps using an ORCA ER (IEEE1394 I/F) digital camera (Hamamatsu, Bridgewater, NJ, USA). Leica Q-Fluoro was used to acquire multifocus images for each of the DAPI, SA, SG and SO optical filters. Ten to 16 images were acquired and signal enumeration was performed on these digital images for 208-641 nuclei for each case. The counted signals were listed and evaluated in Excel-based customised software.

Nuclei that could not be evaluated (e.g., because of insufficient hybridisation or overlapping nuclei) were excluded from further analysis. The results for all 'countable' nuclei were registered in relocation charts in the form of patterns for the entire probe panel. For example, the pattern (2-3-3) refers to two signals for CEP7, three signals for CEP3 and three signals for TERC in a given nucleus. Nuclei with normal signal numbers for the three probes (i.e., 2-2-2) were recorded as 'diploid', and nuclei with four signals for each probe (pattern 4-4-4) were considered 'tetraploid'. The background level for the CEP7-CEP3-TERC probe panel was previously evaluated on cytological slides that contained nuclei from normal cervical cells (Heselmeyer-Haddad et al, 2003). This confirmed that deviation from the expected $(2-2-2)$ pattern was seen in less than $2 \%$ of normal cells.

\section{RESULTS}

\section{Frequent gain of TERC in cervical adenocarcinomas}

Twelve cervical adenocarcinomas (Table 1) were evaluated for copy number changes of the TERC locus at chromosomal band 3 q26 using interphase FISH. The three-colour probe panel consisting of CEP7-CEP3-TERC was simultaneously hybridised to cytospin slides with interphase nuclei prepared from formalinfixed tissue sections. All cases were successfully hybridised and analysed, whereby 208-641 nuclei per case were scored. Figure 1 shows representative hybridisations. Both normal 'diploid' patterns (2-2-2) and nuclei with nondiploid patterns were observed. The results are summarised in Table 2. In all 12 tumours, a significant proportion of 'nondiploid' nuclei was detected, and nine of the tumours exhibited $>50 \%$ 'nondiploid' nuclei. In the individual tumours, nuclei with $>2$ TERC signals were found in similar frequencies as cells with 'nondiploid' pattern, and almost all nuclei with 'nondiploid' pattern exhibited $>2$ TERC signals. In the 'nondiploid' nuclei, a 'tetraploid' pattern (4-4-4) was not commonly observed. In 10 of the tumours, less than $1 \%$ of the nuclei exhibited (4-4-4) and in two cases (4-4-4) was seen in 4 and $12 \%$, respectively (Table 2; exemplified for case 3 in Figure 2). Thus, gain of TERC was present in all tumours studied and was not an effect of 'tetraploid' status.

\section{Intratumour heterogeneity for gain and amplification of the TERC locus}

We observed different FISH patterns in the individual cases. For several of the tumours, one or a few predominating patterns were seen (exemplified for cases 1, 3 and 12 in Figure 2). In addition, all cases exhibited varying proportions of different but recurrent 'nondiploid' patterns. Comparison of signal numbers recorded for TERC vs CEP7 and CEP3 showed increased relative TERC copies in the 12 cases studied (range 1.04-2.0; Table 2). The absolute mean number of TERC signals per nuclei ranged between 2.3 (case 11) and 5.2 (case 10), and in five of the tumours nuclei with more than 10 signals were repeatedly encountered (Table 2). Figure 1 illustrates nuclei of case 10 with amplifications of up to 20 TERC signals and more, together with 5-6 signals for CEP3 and CEP7 (Figure $1 \mathrm{G}$ and $\mathrm{H}$ ), which shows that TERC can be highly amplified in certain cases. However, the more common pattern observed in most cases are low-level copy numbers of TERC, often on a still diploid background with patterns that can be reconciled with extra chromosomes of 3 or an isochromosome $3 q$ formation.

\section{Gain of TERC and HPV infection}

The clinical and follow-up information as well as HPV status of the 12 cervical adenocarcinomas are provided in Table 1. Eight cases were HPV-positive and in the other four HPV was negative. Very high proportions of 'nondiploid' nuclei with $>2$ TERC signals were demonstrated in HPV-positive (83-99\%) as well as HPVnegative (86-99\%) tumours. The absolute numbers of TERC signals per nucleus were similarly increased in HPV-positive (mean 3.4; range 2-22) and HPV-negative (mean 3.3; range 2-12) tumours. No apparent associations between TERC copy numbers and clinical characteristics, such as age at diagnosis, stage, differentiation, lymph node involvement or outcome at followup, were noted. Thus, gain of TERC is characteristic of cervical adenocarcinomas per se.

\section{DISCUSSION}

In our study, we analysed a series of 12 primary cervical adenocarcinomas for gain or amplification of the human telomerase gene TERC, which maps to chromosome band $3 \mathrm{q} 26$. Eight of the 12 carcinomas (67\%) were HPV-positive (five tumours were positive for HPV 18, two for HPV 16 and one for HPV 45) (Skyldberg et al, 1999; Andersson et al, 2003a). We then used FISH with a custom-designed probe panel that includes the human telomerase gene (TERC) to assess the potential of this genetic marker to ameliorate the morphological diagnosis of cervical adenocarcinomas. This is the first study that reports the consistent gain of the human telomerase gene TERC in cervical adenocarcinomas. 


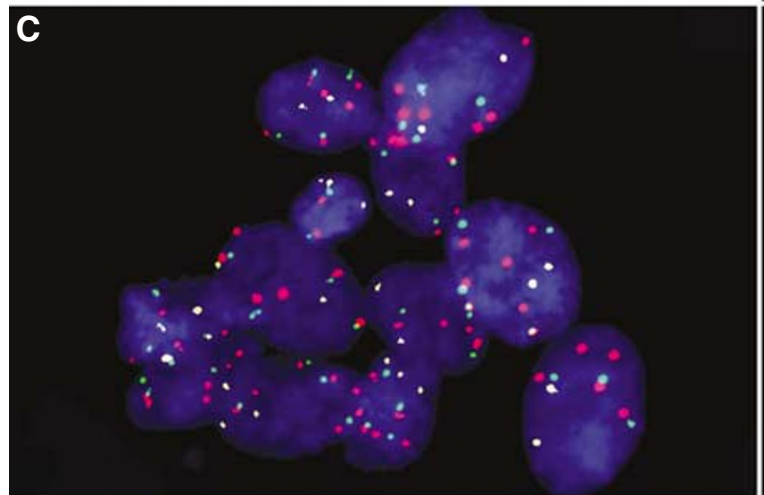

$\mathbf{E}$
A

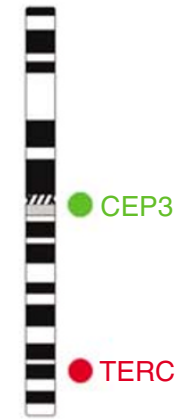

3

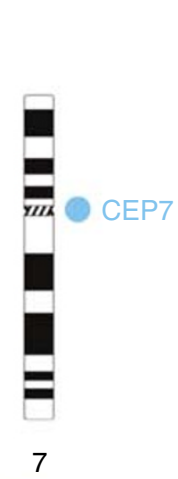

B

D

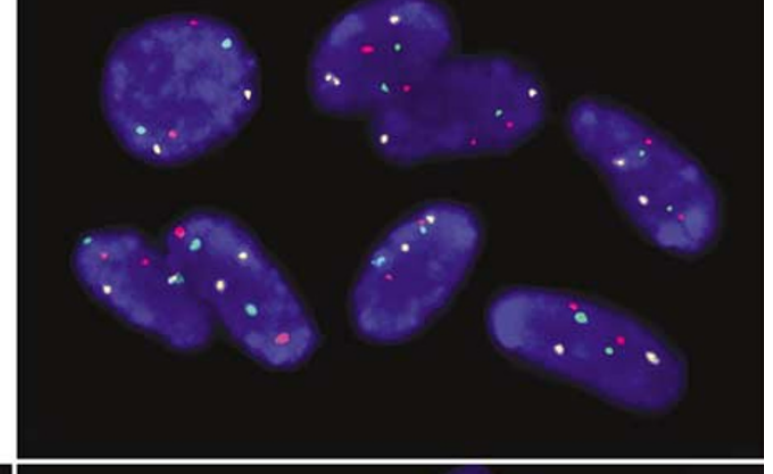

.

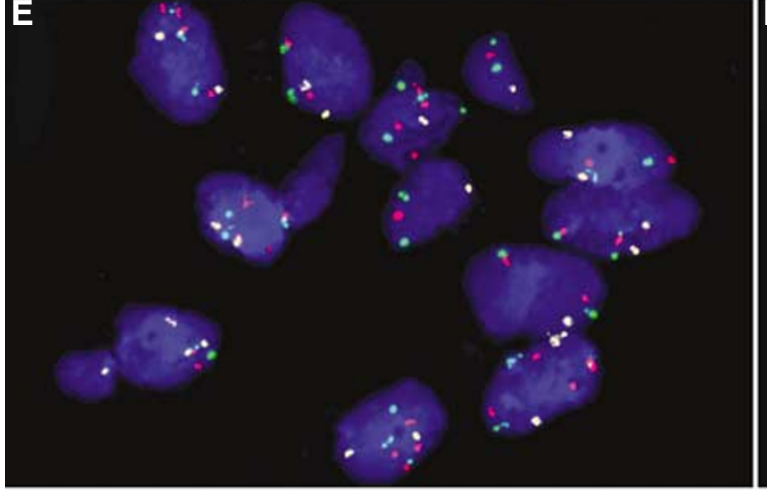

F

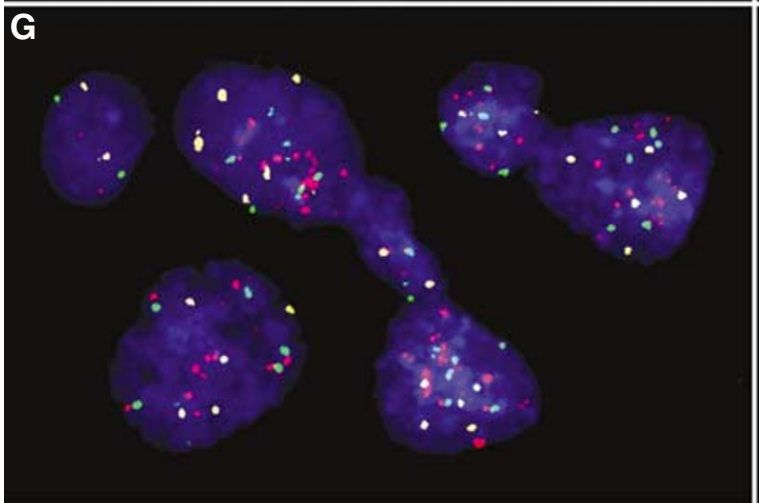

$\mathbf{H}$

Figure I Interphase FISH analysis showing gain of TERC in 3q26 in cervical adenocarcinomas. (A) Chromosomal location of the triple-colour probe set: CEP 7 (labelled with Spectrum Aqua, which is pseudocoloured in yellowish-white in panels B, C, E and G), CEP 3 (Spectrum Green, pseudocoloured in green in panels B, C, E and G), and TERC (Spectrum Orange, pseudocoloured in red). (B) Normal nuclei with the expected pattern of two signals per probe per nucleus (2-2-2). (C and D) HPV-negative case number 4, (E and $\mathbf{F})$ HPV-infected case number 6 and $(\mathbf{G}$ and $\mathbf{H})$ HPV-positive case number I0. All cases exhibit varying extents of gain of TERC. The cell clusters in $\mathbf{C}, \mathbf{E}$ and $\mathbf{G}$ and in $\mathbf{D}, \mathbf{F}$ and $\mathbf{H}$ show identical cells with either the triple-colour probe set shown or with the TERC probe only, respectively.

Yang et al (2001) analysed 20 cases of cervical adenocarcinomas with CGH and found that genetic alterations were evident in most of the cervical adenocarcinomas, including the gain of $3 \mathrm{q}$, which was detected in $70 \%$ of these 20 tumours. From the findings of similar $3 \mathrm{q}$ amplifications in adenocarcinomas as reported for squamous cell carcinoma, it was proposed that similar 


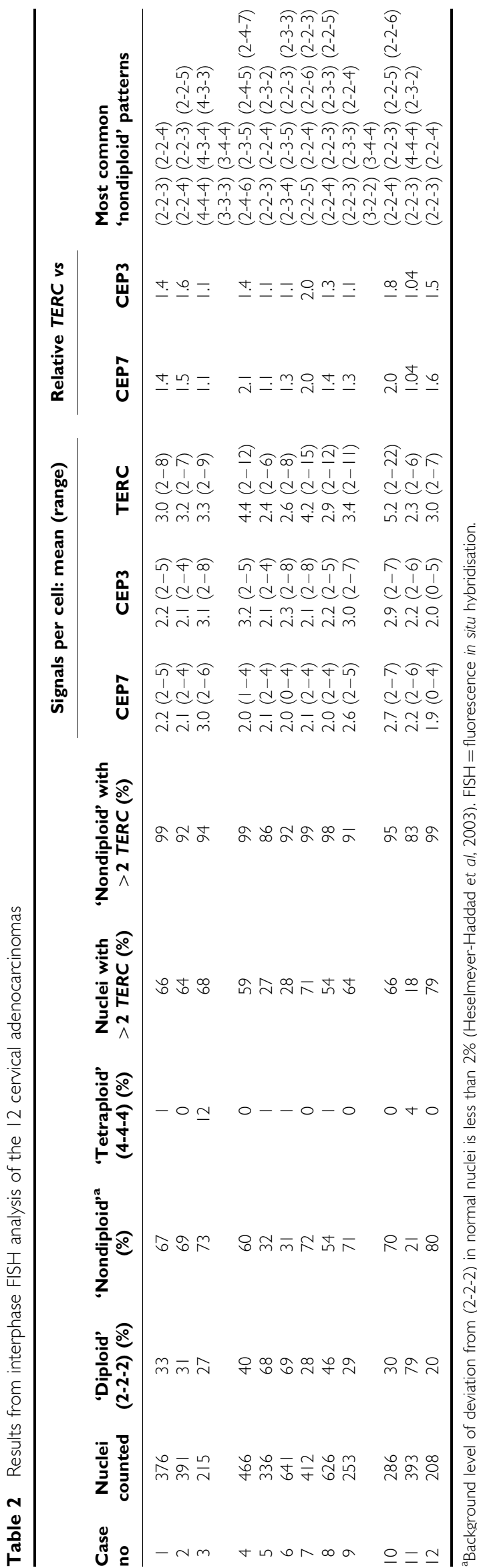

mechanisms are involved in the development of the two types of cervical cancer (Yang et al, 2001). In our study, using FISH we detected a gain of $3 \mathrm{q}$ in all our cases. This difference is likely attributable to the different sensitivity of the respective techniques. Detection of a trisomy by CGH requires this numerical aberration to be present in at least $40 \%$ of the cells. Fluorescence in situ hybridisation, of course, detects changes on a single-cell basis and is therefore not sensitive to dilution. Additionally, small regional low-copy number amplicons might escape detection by CGH.

In our study, HPV infection was not detected in four of the cases, which could either reflect true HPV negativity or represent 'false negatives' owing to technical difficulties in HPV genome detection. In the current literature, HPV infection has not been reported in the same proportion in adenocarcinomas as in squamous cervical carcinomas (Tenti et al, 1996; Skyldberg et al, 1999; Andersson et al, 2003b; Clifford et al, 2003; Zielinski et al, 2004; Castellsague et al, 2006). Perhaps, oncogenic factors other than HPV are more likely to play a role in the malignant transformation of cervical adenocarcinomas (Skyldberg et al, 1999; Pirog et al, 2000).

The application of screening programmes has reduced the incidence of squamous carcinoma in Sweden substantially during the last decades (Mählck et al, 1994; Ponten et al, 1995). Yet, several studies suggest that a single Pap smear has a high falsenegative rate (Macgregor et al, 1994). A review of evidence-based data revealed that as many as $50 \%$ of precancerous cervical lesions may be missed by a single Pap test (Sherman et al, 1994). Kinney et al (1998) showed that only $30 \%$ of women with histopathologically confirmed high-grade disease had corresponding atypia in their Pap smears. Against this background, a new screening strategy for cervical cancer has been presented in which HPV testing is combined with cytological examinations (Walboomers et al, 1999). However, screening methods currently utilised to detect preinvasive and early invasive squamous cervical cancer had only a limited effect in detecting precursor lesions of cervical adenocarcinoma.

Hildesheim et al (1999) classified women as having rapid-onset cervical cancer with the following criteria: a detection of cervical cancer within 5 years of at least one normal Pap smear or within 10 years after the first sexual intercourse. Our data showing that 10 of the 12 women had a normal Pap smear within 3 years before the diagnosis of invasive disease may support the hypothesis of a rapid-onset carcinoma. On the other hand, as the sensitivity of a Pap smear for the detection of cervical adenocarcinomas is very low (Krane et al, 2001), the rapid-onset hypothesis may not apply to our cases.

Cervical squamous cell carcinomas are defined by a nonrandom and recurrent distribution of genomic imbalances. In addition to $\mathrm{HPV}$, the sequential transformation of cervical squamous epithelium requires the acquisition of additional copies of chromosome arm 3q. In the current study, we wanted to analyse and understand whether the gain of $3 \mathrm{q}$ plays a similar role in the tumorigenesis of cervical adenocarcinomas. Using a genomic probe for the TERC gene on chromosome band $3 \mathrm{q} 26$ in combination with two centromere-specific probes (CEP3 and CEP7), we showed high copy numbers of this locus in cervical adenocarcinomas. Gain or amplification of $3 \mathrm{q}$ was found in all cervical adenocarcinomas investigated. Therefore, the application of this probe set may provide an objective genetic test for the diagnosis of cervical adenocarcinomas and might assist in the interpretation of smears with a high degree of glandular cells.

Heselmeyer-Haddad et al (2003) showed in a series of Pap smears of cervical precursor lesions of squamous cell origin that analysis of the TERC gene amplification is able to distinguish normal epithelium and low-grade dysplasia from the group of high-grade lesions with high specificity and sensitivity. In another paper, the same group showed that gain of TERC was found in $33 \%$ of cytological normal Pap smears from women who developed 

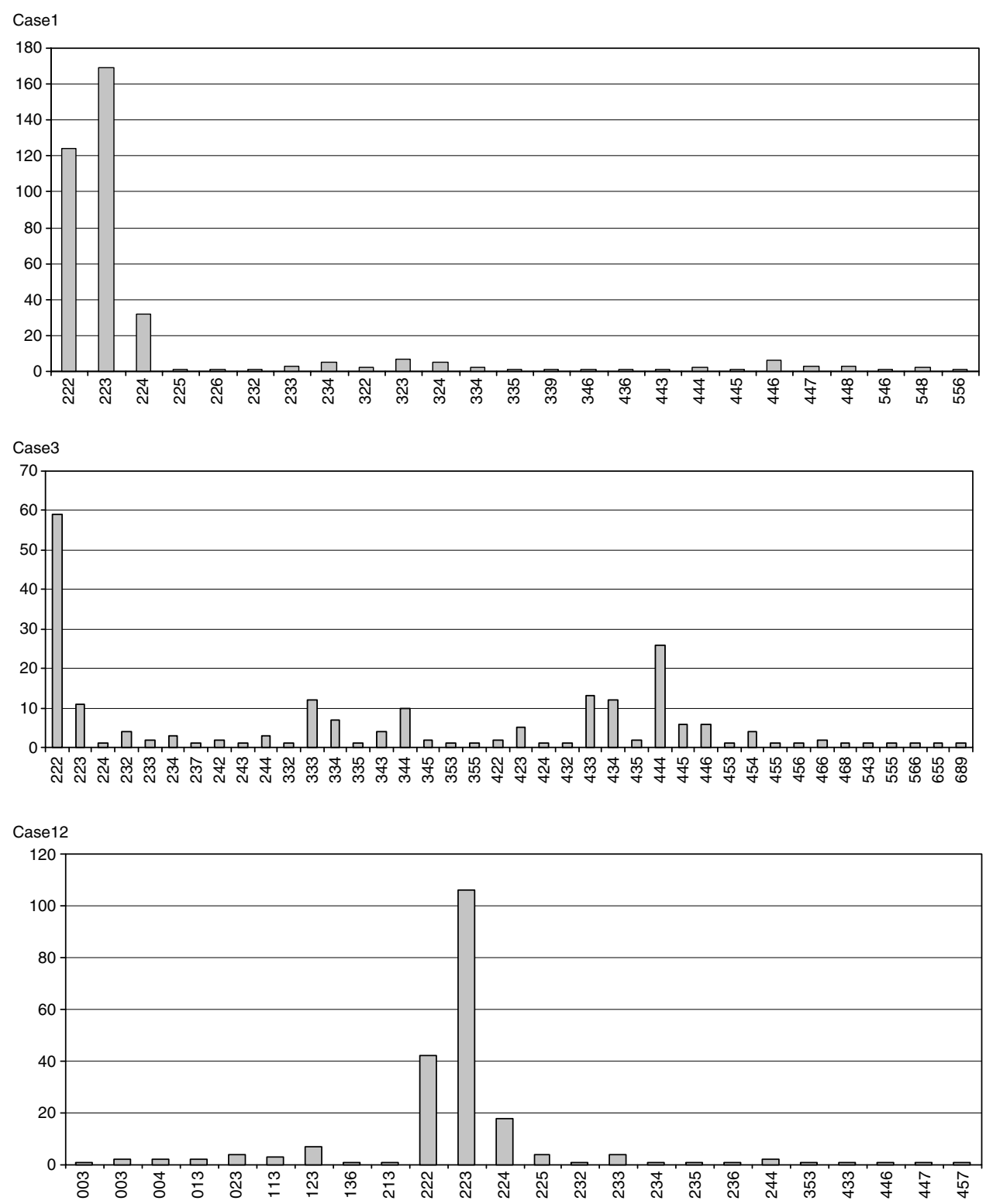

Figure 2 Diagrams illustrating the scoring results from interphase FISH analyses in cases I, 3 and I2. Scoring patterns for CEP3-CEP7-TERC are given along the $x$ axis and the numbers of detected nuclei with a given pattern are given on the $y$ axis.

carcinoma in situ or invasive cervical squamous cancer in the future (Heselmeyer-Haddad et al, 2005). In the same paper, the authors were also able to show that all CIN1/CIN2 lesions with a diploid pattern for the TERC probe panel (CEP7-CEP3-TERC) eventually regressed to normal Pap smears, whereas the CIN1/ CIN2 lesions with TERC amplification progressed. Lesions with a tetraploid pattern for the panel (four signals for each probe) could develop either way. The author concluded that the detection of gain and amplification of TERC in routinely collected Pap smears can assist in identifying lesions with a high progression risk. As the results of the current study indicate, TERC appears to play a similar important role in the carcinogenesis of cervical adenocarcinomas. Interestingly, even the patterns of gain found in the adenocarcinomas are very comparable to the patterns found in cervical dysplasias of squamous cell type (Heselmeyer-Haddad et al, 2003, 2005). The most common patterns observed for both of the cell types are low-level gains of TERC on a diploid background (3-5 signals of TERC). Given the similarity to the results of the studies of TERC in cervical squamous cell lesions, it seems to be a distinct possibility that analysis of TERC gain may also help to find women at risk for developing adenocarcinoma. An objective molecular marker in this patient group is of extreme importance because of the cytomorphologically difficult identification of these cells and the limited numbers of representative cells owing to technical problems in sampling these lesions. As shown in our study, many women with cervical adenocarcinoma presented with morphologically normal Pap smears, often only $1-2$ years before diagnosis. A marker like TERC could have considerable impact on the detection of cervical adenocarcinomas at an earlier stage. Fluorescence in situ hybridisation analyses of precursor lesions, that is, adenocarcinomas in situ and dysplasias will be the next step to verify the potential of TERC as a marker of early detection and progression in cervical adenocarcinomas. We are therefore planning to conduct both retrospective studies with archival Pap smears and prospective randomised studies with routinely collected Pap smears. 


\section{ACKNOWLEDGEMENTS}

We wish to express our gratitude to Buddy Chen and Joseph Cheng for IT support and manuscript editing, and Juan Castro, Inga Maurin, Nicole White and Diana Tong for excellent technical assistance. This study was supported by the Swedish Cancer Foundation, Swedish Research Council, Medical Research Council, Göran Gustafsson Foundation, Cancer Society in Stockholm, Stockholm County Council, NIH and Swedish Labour Market Insurance.

\section{REFERENCES}

Andersson S, Larson B, Hjerpe A, Silfversward C, Sällström J, Wilander E, Rylander E (2003a) Adenocarcinoma of the uterine cervix: the presence of human papillomavirus and the method of detection. Acta Obstet Gynecol Scand 82: 960 -965

Andersson S, Rylander E, Larson B, Sigurdardottir S, Backlund I, Sällström J, Wilander E (2003b) Types of human papillomavirus revealed in cervical adenocarcinomas after DNA sequencing. Oncol Rep 10: $175-179$

Benedet JL, Bender H, Jones III H, Ngan HY, Pecorelli S (2000) FIGO staging classifications and clinical practice guidelines in the management of gynecologic cancers. FIGO Committee on Gynecologic Oncology. Int $J$ Gynaecol Obstet 70: 209-262

Bergström R, Sparen P, Adami HO (1999) Trends in cancer of the cervix uteri in Sweden following cytological screening. Br J Cancer 81: 159-166

Bittner ML, Morrison LE, Legator MS (1996) Direct label transaminated DNA probe compositions for chromosome identification and methods for their manufacture. United States Government Printing Office, Washington, DC. United States Patent 5, 491, 224

Bosch FX, Manos MM, Munoz N, Sherman M, Jansen AM, Peto J, Schiffman MH, Moreno V, Kurman R, Shah KV (1995) Prevalence of human papillomavirus in cervical cancer: a worldwide perspective. International Biological Study on Cervical Cancer (IBSCC) Study Group. J Natl Cancer Inst 87: 796-802

Bosch FX, Munoz N (2002) The viral etiology of cervical cancer. Virus Res 89: $183-189$

Castellsague X, Díaz M, de Sanjosé S, Muñoz N, Herrero R, Franceschi S, Peeling RW, Ashley R, Smith JS, Snijders PJF, Meijer CJLM, Bosch FX (2006) Worldwide human papillomavirus etiology of cervical adenocarcinoma and its cofactors: implications for screening and prevention. J Natl Cancer Inst 98: 303-315

Castro J, Heiden T, Wang N, Tribukait B (1993) Preparation of cell nuclei from fresh tissues for high-quality DNA flow cytometry. Cytometry 14: $793-804$

Clifford GM, Smith JS, Aguado T, Franceschi S (2003) Comparison of HPV type distribution in high-grade cervical lesions and cervical cancer: a meta-analysis. Br J Cancer 89: $101-105$

Dahlgren L, Mellin H, Wangsa D, Heselmeyer-Haddad K, Bjornestal L, Lindholm J, Munck-Wikland E, Auer G, Ried T, Dalianis T (2003) Comparative genomic hybridization analysis of tonsillar cancer reveals a different pattern of genomic imbalances in human papillomaviruspositive and -negative tumors. Int J Cancer 107: 244-249

Heselmeyer K, du Manoir S, Blegen H, Friberg B, Svensson C, Schrock E, Veldman T, Shah K, Auer G, Ried T (1997b) A recurrent pattern of chromosomal aberrations and immunophenotypic appearance defines anal squamous cell carcinomas. Br J Cancer 76: 1271-1278

Heselmeyer K, Macville M, Schrock E, Blegen H, Hellstrom AC, Shah K, Auer G, Ried T (1997a) Advanced-stage cervical carcinomas are defined by a recurrent pattern of chromosomal aberrations revealing high genetic instability and a consistent gain of chromosome arm 3q. Genes Chromosomes Cancer 19: 233-240

Heselmeyer K, Schrock E, du Manoir S, Blegen H, Shah K, Steinbeck R, Auer G, Ried T (1996) Gain of chromosome 3q defines the transition from severe dysplasia to invasive carcinoma of the uterine cervix. Proc Natl Acad Sci USA 93: 479-484

Heselmeyer-Haddad K, Janz V, Castle PE, Chaudhri N, White N, Wilber K, Morrison LE, Auer G, Burroughs FH, Sherman ME, Ried T (2003) Detection of genomic amplification of the human telomerase gene (TERC) in cytologic specimens as a genetic test for the diagnosis of cervical dysplasia. Am J Pathol 163: 1405-1416

Heselmeyer-Haddad K, Sommerfeld K, White NM, Chaudhri N, Morrison LE, Palanisamy N, Wang ZY, Auer G, Steinberg W, Ried T (2005) Genomic amplification of the human telomerase gene (TERC) in Pap smears predicts the development of cervical cancer. Am J Pathol 166: $1229-1238$

Hildesheim A, Hadjimichael O, Schwartz PE, Wheeler CM, Barnes W, Lowell DM, Willett J, Schiffman M (1999) Risk factors for rapid-onset cervical cancer. Am J Obstet Gynecol 180: 571-577

Kinney WK, Manos MM, Hurley LB, Ransley JE (1998) Where's the highgrade cervical neoplasia? The importance of minimally abnormal Papanicolaou diagnoses. Obstet Gynecol 91: 973-976

Krane JF, Granter SR, Trask CE, Hogan CL, Lee KR (2001) Papanicolaou smear sensitivity for the detection of adenocarcinoma of the cervix: a study of 49 cases. Cancer 93: 8-15

Macgregor JE, Campbell MK, Mann EM, Swanson KY (1994) Screening for cervical intraepithelial neoplasia in north east Scotland shows fall in incidence and mortality from invasive cancer with concomitant rise in preinvasive disease. $B M J$ 308: $1407-1411$

Mählck CG, Jonsson H, Lenner P (1994) Pap smear screening and changes in cervical cancer mortality in Sweden. Int J Gynecol Obstet 44: $267-272$

McCluggage WG, Sumathi VP, McBride HA, Patterson A (2002) A panel of immunohistochemical stains, including carcinoembryonic antigen, vimentin, and estrogen receptor, aids the distinction between primary endometrial and endocervical adenocarcinomas. Int J Gynecol Pathol 21: $11-15$

Parkin DM, Bray F, Ferlay J, Pisani P (2001) Estimating the world cancer burden: Globocan 2000. Int J Cancer 94: 153-156

Pirog EC, Kleter B, Olgac S, Bobkiewicz P, Lindeman J, Quint WG, Richart RM, Isacson C (2000) Prevalence of human papillomavirus DNA in different histological subtypes of cervical adenocarcinoma. Am J Pathol 157: $1055-1062$

Ponten J, Adami HO, Friberg LG, Gustafsson L, Miller AB, Parkin M, Sparen P, Trichopoulos D (1995) HPV and cervical cancer. Int J Cancer 63: 317

Sasieni P, Adams J (2001) Changing rates of adenocarcinoma and adenosquamous carcinoma of the cervix in England. Lancet 357: 1490 - 1493

Sherman ME, Schiffman MH, Lorincz AT, Manos MM, Scott DR, Kuman RJ, Kiviat NB, Stoler M, Glass AG, Rush BB (1994) Toward objective quality assurance in cervical cytopathology:correlation of cytopathologic diagnosis with detection of high-risk human papillomavirus types. Am J Clin Pathol 102: $182-187$

Skyldberg BM, Murray E, Lambkin H, Kelehan P, Auer GU (1999) Adenocarcinoma of the uterine cervix in Ireland and Sweden: human papillomavirus infection and biologic alterations. Mod Pathol 12: $675-682$

Smith HO, Tiffany MF, Qualls CR, Key CR (2000) The rising incidence of adenocarcinoma relative to squamous cell carcinoma of the uterine cervix in the United States - a 24-year population-based study. Gynecol Oncol 78: $97-105$

Stoltzfus P, Heselmeyer-Haddad K, Castro J, White N, Silfverswärd C, Sjövall K, Einhorn N, Tryggvason K, Auer G, Ried T, Nordström B (2005) Gain of chromosome $3 q$ is an early and consistent genetic aberration in carcinomas of the vulva. Int J Gynecol Cancer 15: $120-126$

Tenti P, Romagnoli S, Silini E, Zappatore R, Spinillo A, Giunta P, Cappellini A, Vesentini N, Zara C, Carnevali L (1996) Human papillomavirus types 16 and 18 infection in infiltrating adenocarcinoma of the cervix: PCR analysis of 138 cases and correlation with histologic type and grade. Am J Clin Pathol 106: $52-56$

Vizcaino AP, Moreno V, Bosch FX, Munoz N, Barros-Dios XM, Parkin DM (1998) International trends in the incidence of cervical cancer: I. Adenocarcinoma and adenosquamous cell carcinomas. Int J Cancer 75: $536-545$ 


\section{Gain of TERC in cervical adenocarcinomas}

$S$ Andersson et al

Walboomers JM, Jacobs MV, Manos MM, Bosch FX, Kummer JA, Shah KV, Snijders PJ, Peto J, Meijer CJ, Munoz N (1999) Human papillomavirus is a necessary cause of invasive cervical cancer worldwide. J Pathol 189: 12 - 19

Yang YC, Shyong WY, Chang MS, Chen YJ, Lin CH, Huang ZD, Wang, Hsu MT, Chen ML (2001) Frequent gain of copy number on the long arm of chromosome 3 in human cervical adenocarcinoma. Cancer Genet Cytogenet 131: $48-53$
Zielinski GD, Snijders PJF, Rozendaal L, Daalmeijer NF, Risse EKJ, Voorhorst FJ, Jiwa NM, van der Linden HC, de Schipper FA, Runsink AP, Meijer CJLM (2004) The presence of high-risk HPV combined with specific p53 and p16INK4a expression patterns points to high-risk HPV as the main causative agent for adenocarcinoma in situ and adenocarcinoma of the cervix. J Pathol 201: $535-543$ 